\title{
Estudios de actividad radical en lima ácida común (Citrus aurantifolia) usando ${ }^{32} \mathrm{P}$
}

\section{Studies of the root activity in common acid lime (Citrus aurantifolia) using ${ }^{32} \mathrm{P}$}

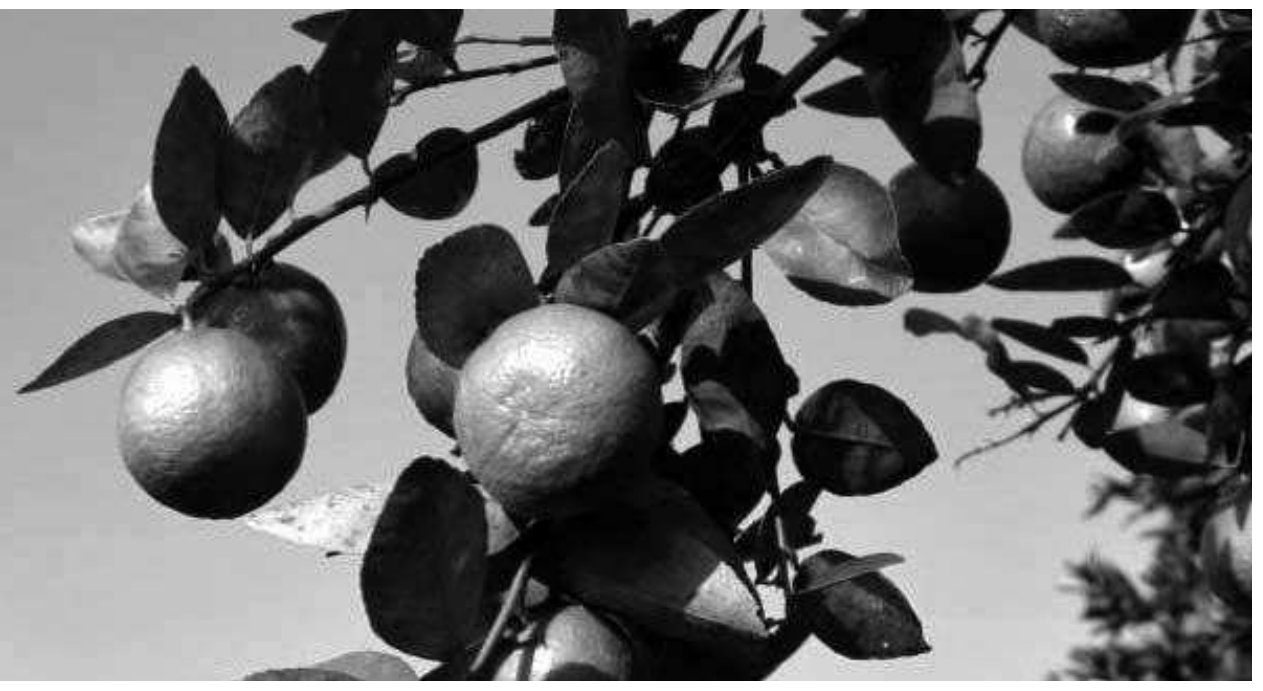

MARÍA ELENA BÁEZ1

CONRADO DELGADO²

DIEGO MIRANDA ${ }^{3}$

ROBERTO OBANDO 4

Árbol de lima ácida común en fructificación.

Foto: J. Orduz

\section{RESUMEN}

Puesto que el sistema radical es responsable de la toma de agua y nutrientes desde el suelo, el objetivo de este trabajo fue determinar la actividad radical en plantas de lima ácida común (sobre el patrón Limón común), de cinco años de edad, mediante la utilización del isótopo ${ }^{32} \mathrm{P}$ como trazador. En Flandes, Tolima (Colombia), a $28^{\circ} \mathrm{C}$ temperatura y $68 \% \mathrm{HR}, 1.177 \mathrm{~mm}$ precipitación/año, en un suelo franco arenoso, se evaluaron tres profundidades de aplicación del ${ }^{32} \mathrm{P}$ 15, 30 y $45 \mathrm{~cm}$ y tres distancias desde el tronco 100, 150 y $200 \mathrm{~cm}$. La solución isotópica se aplicó en dosis de $5 \mathrm{~mL}$ ubicados alrededor de la zona de plateo del árbol. La unidad experimental fue un árbol y se empleó una técnica de muestreo estándar para hoja, ramas (tallo) y frutos con un intervalo de muestreo de 15 días y un total de seis muestreos. La actividad del isótopo en las muestras se determinó por conteo en un contador de centelleo líquido por efecto Cerenkov. La mayor actividad de las raíces se presentó en los primeros $15 \mathrm{~cm}$ y entre $15-30 \mathrm{~cm}$ de profundidad, alcanzando valores entre 14,5 y $18 \%$. La distancia a la cual se encontró el mayor porcentaje de actividad fue a $100 \mathrm{~cm}$ y hasta los $150 \mathrm{~cm}$ desde el tronco. La estructura de la planta donde se logró hacer mayor detección del isótopo fue la hoja. Esta información indica que para árboles de lima ácida común de cinco años las raíces más activas se ubican en los primeros $30 \mathrm{~cm}$ de profundidad y entre 100 y $150 \mathrm{~cm}$ de distancia desde el tronco, por lo tanto es en esta zona donde deben ubicarse los fertilizantes y el suministro de agua para el cultivo y no ubicar arreglos de cultivos múltiples.

Grupo Nutricional Vegetal, Instituto de Ciencias Nucleares y Energías Alternativas (INEA), Bogotá.

Grupo de Agricultura, Instituto de Ciencias Nucleares y Energías Alternativas (INEA), Bogotá.

Profesor Asociado, Facultad de Agronomía, Universidad Nacional de Colombia, Bogotá. dmirandal@unal.edu.co

Grupo de Agricultura, Instituto de Ciencias Nucleares y Energías Alternativas (INEA), Bogotá. 


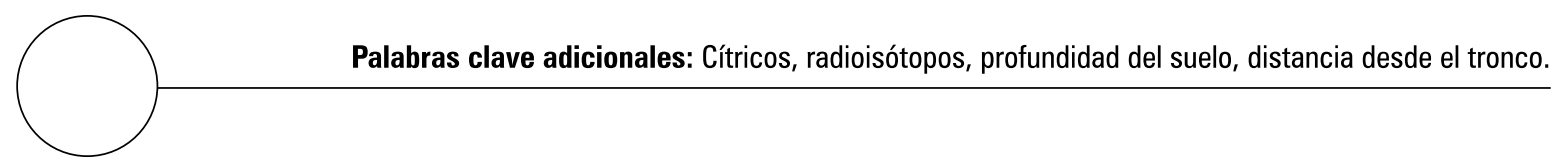

\section{ABSTRACT}

Since the root system is responsible for water and nutrient absorption from soil, the objective of this research was to determine the root activity of five year old plants of common acid lime on Common Lime rootstock using isotope ${ }^{32} \mathrm{P}$ as a tracer. In Flandes, Tolima, Colombia $\left(28^{\circ} \mathrm{C}\right.$ mean temperature, $68 \% \mathrm{RH}, 1.177 \mathrm{~mm}$ annual precipitation, and sandy-loam soil), the application of $32 \mathrm{P}$ on three soil depths $(15,30$, and $45 \mathrm{~cm})$ and distances from the stem $(100,150$, and $200 \mathrm{~cm})$ was evaluated. The isotopic solution at a rate of $5 \mathrm{~mL}$ was applied to the zone of tree disc. The experimental unit was one tree and a standard sampling was used for leaves, branches (stem), and fruits with a 15 day sampling interval in six samplings. The isotope activity in the samples was determined by a counter of liquid sparkle by Cerenkov method. The highest root activity appeared in the first $15 \mathrm{~cm}$ and between $15-30 \mathrm{~cm}$ soil depth, reaching values between 14.5 and $18 \%$. The distance from the stem, where highest percentage of activity was found, lay at $100 \mathrm{~cm}$ and up to $150 \mathrm{~cm}$ from the stem. The structure of the plant, where a major detection of the isotope was achieved, was the leaf and, secondly, the root. This information indicates that, for five year old trees of common acid lime, the most active roots are located in the first $30 \mathrm{~cm}$ of soil and between 100 and $150 \mathrm{~cm}$ of distance from the stem. Therefore, it is this zone, where the fertilizers and water should be supplied and multiple cultures may not be located.

Additional key words: Citrus, radioisotopes, soil depth, distance from the stem.
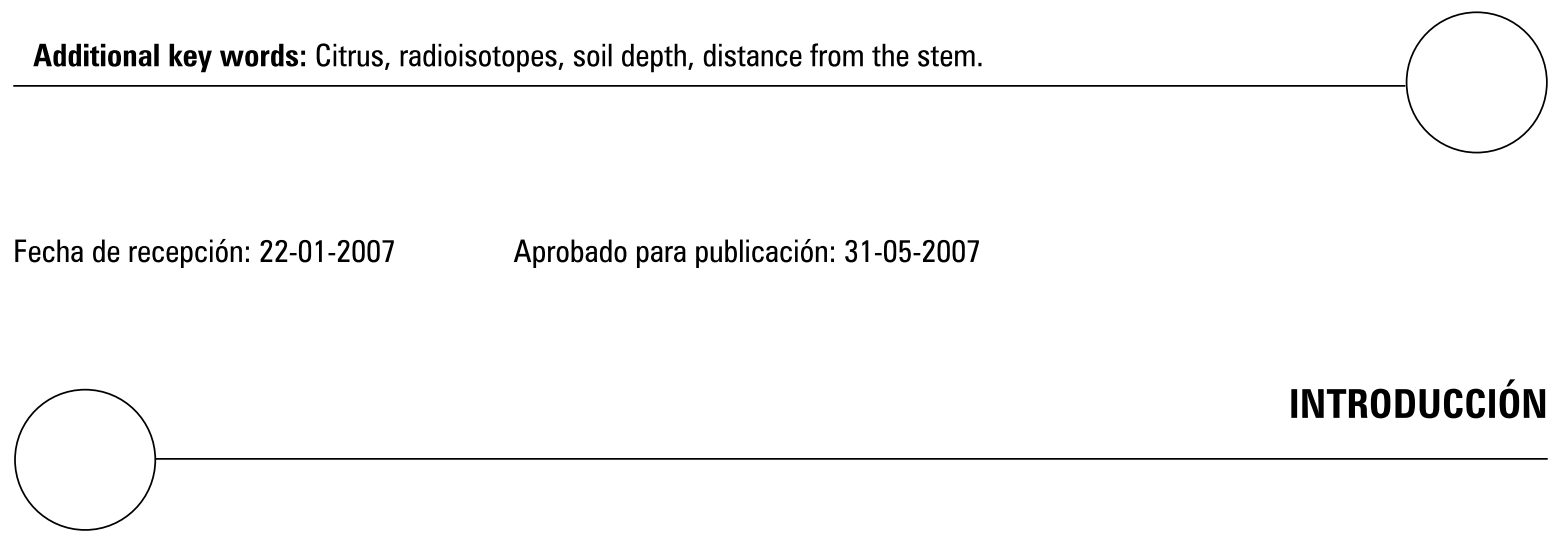

INTRODUCCIÓN

Según la FAO, en 2001, se cosecharon en el mundo 742.027 ha de limas y limones. Los principales productores son: México, India, Brasil, Irán y España, países que en conjunto concentraron el 51,3\% del área cosechada a nivel mundial. Las mayores tasas de crecimiento, tanto del área cosechada como de la producción, se registraron en Colombia, México, India, Irán, China y Argentina donde el crecimiento de la producción superó el del área cosechada, lo que refleja un aumento en los rendimientos. La producción de limones de Colombia creció, entre 1992 y 2001, 26\% anual, pero el volumen no alcanzó las 100.000 ton, los rendimientos promedio fueron de 16,07 tha ${ }^{-1}$, superior a la de México (principal productor). La producción de cítricos en Colombia representa el $12 \%$ de la producción hortofrutícola nacional y el $8 \%$ del área cosechada; dentro del sector de los cítricos, las limas y limones representan el 9\% de la producción. Según información del Ministerio de Agricultura y Desarrollo Rural, en el 2001 se cosecharon 4.740 ha de limas y limones en Colombia (CCI, 2002).

La raíz de los cítricos es sólida, blanca y, bajo condiciones de cultivo, posee gran cantidad de 
pelos radicales (Agusti, 2003). La raíz sirve para anclar la planta en el suelo, para absorber agua y nutrientes, para producir parte de los aminoácidos y para excretar sustancias o metabolitos de desecho, pero que sirven de todos modos de sustento a los microorganismos en la rizósfera, y que movilizan nutrientes (Delgado, 1995). La raíz no puede absorber en toda su superficie y si lo hace es en mínima cantidad. El lugar principal de absorción está inmediatamente después de la punta de las radiculas, en la zona donde ya se forman pelos y donde la cáscara todavía no esta suberificada (Epstein y Bloom, 2005). Otras funciones importantes de las raíces son la síntesis de hormonas (citoquininas y giberelinas, principalmente), el intercambio gaseoso con la rizósfera y el almacenamiento de sustancias de reserva (Fischer, 1994).

Las raíces crecen tanto en longitud como en diámetro, en longitud por la división celular y alargamiento de los tejido primarios, y en diámetro como un resultado de la actividad meristemática secundaria en el cambium lateral y del corcho, penetrando al suelo debido a las células en la región de elongación, las cuales se extienden unas cuantos mm detrás de la caliptra, se alargan y empujan el ápice meristemático todavía no diferenciado hacia adelante (Ryugo, 1993). La frecuencia y distribución de la formación de raíces laterales controla en parte la forma global del sistema radical y por tanto las zonas del suelo que se exploran (Salisbury y Ross, 1994).

La respuesta a la fertilización fosfórica en suelos con cierto nivel de fósforo tiende a ser diferente en suelos arenosos que en suelos con mayores contenidos de limos y arcillas. La difusión es el proceso más importante del movimiento del fósforo hacia las raíces y es lento en suelos de textura granular, donde se requieren altas tasas de fertilización. Algunos componentes del suelo reaccionan en realidad con el fertilizante y disminuyen su disponibilidad. Esto hace que sean mayores las cantidades necesarias a aplicar en esos suelos en orden a aumentar la disponibilidad del fósforo para la planta (Delgado, 1995).

La metodología de trazadores consiste básicamente en inyectar un isótopo adecuado al suelo o a la planta. En el procedimiento de estas técnicas con trazadores isotópicos se han adoptado dos procedimientos como son: (a) inyección de un isótopo como ${ }^{32} \mathrm{P}$ o ${ }^{86} \mathrm{Rb}$ en el tallo de la planta tomando muestras del suelo-raíz y midiendo la radiactividad; (b) la inyección al suelo con una solución de fosfato marcado con ${ }^{32} \mathrm{P}$ en diferentes puntos (distancias y profundidades) y al realizar la medición de la radiactividad de las muestras vegetales se comprueba la actividad de las raíces en esos puntos (OIEA, 1975). Ademas el uso de trazadores $\left({ }^{32} \mathrm{P},{ }^{33} \mathrm{P},{ }^{15} \mathrm{~N}\right)$ permite monitorear el momento y la localización de un compuesto en el sistema suelo-planta-agua (Pino, 2005) y determinar la cantidad de nutriente que absorbe la planta y la que se pierde en el medio ambiente (Quezada, 2006). El empleo de ${ }^{32} \mathrm{P}$, emisor $\beta^{-}$con 14,29 días de vida media, resulta apropiado para este tipo de estudio por ser un nutriente fácilmente asimilable por los vegetales y poseer la característica de escasa movilidad en el suelo, lo cual permite una discriminación del espacio con adecuada precisión para este fin (IAEA, 1975).

El ${ }^{32} \mathrm{P}$ se mide en un contador de centello líquido por efecto Cerenkov (López et al., 2000). La técnica de centello líquido ha promovido la aplicación de radionuclidos en ciencias agrícolas y biológicas porque ella permite el uso de emisores de partículas beta de baja energía. Con esta técnica, la muestra para ser contada se coloca en una solución que contiene un centelleador orgánico en un solvente orgánico aromático. Cada átomo o molécula del centelleador reduciéndose en gran parte la autoabsorción (Delgado, 1995). La actividad de un isótopo se expresa comúnmente en cuentas por minuto o en cuentas por segundo (cpm, cps); o si se conoce la eficiencia de conteo, se expresa en desintegraciones por unidad de tiempo (dpm, dps). La actividad depende del 
tamaño de la muestra, por lo cual es conveniente definir una específica para describir la relación del número de átomos radioactivos a átomos no radioactivos del mismo elemento, ya sea a través del peso total o del volumen de la muestra. Así la actividad específica para el isótopo radioactivo se indica en $\mathrm{mCi} / \mathrm{gP}$ (Pino, 2005).

El método directo de utilización de isótopos provee un medio no solo la absorción de nutrientes por la planta sino también la utilización de nutrientes del suelo, el efecto de la fertilización y otras prácticas de manejo sobre la absorción de nutrientes (Pino, 2005). Tiene gran sensibilidad para evaluar las raíces fisiológicamente activas, permitiendo determinar no sólo su presencia sino también su funcionalidad. La información obtenida, de carácter cualitativo y cuantitativo enfatiza la actividad radical, el desarrollo y distribución espacial, independientemente de la masa de raíces, permitiendo además el estudio de sistemas radicales superpuestos en el espacio, por lo que resulta una técnica muy apropiada e indicada en el estudio de competencia en comunidades polifíticas (Bujan et al., 1998).

El objetivo del estudio fue determinar la mayor actividad radicular en lima ácida común (limón común) Citrus aurantifolia usando ${ }^{32} \mathrm{P}$ a diferentes distancias del tronco y profundidades de suelo.

\section{MATERIALES Y MÉTODOS}

En el segundo semestre de 1996, en el municipio de Flandes (Tolima, Colombia) ubicado en el clima cálido seco a una altura de $360 \mathrm{msnm}$, con una temperatura promedio de $28^{\circ} \mathrm{C}$, una humedad relativa del $68 \%$ y una precipitación promedio de $1.177 \mathrm{~mm}$ por año. El suelo es de textura franco arenosa, clasificada con Tepic haplustalf pertenecientes al abanico de Espinal, con características físicas que se muestran en la tabla 1.
Se utilizó un diseño estadístico completamente al azar con arreglo factorial y submuestreo. Se evaluaron tres profundidades de aplicación del isótopo 15,30 y $45 \mathrm{~cm}$ y tres distancias 100 , 150 y $200 \mathrm{~cm}$ en un factorial completo, con un árbol por parcela y 4 repeticiones para un total de 36 árboles de lima ácida común (Limón común) de cinco años de edad sobre el patrón Limón común, con características homogéneas plantados a una distancia de $7 \times 7 \mathrm{~m}$. El volumen de solución radiactiva fue de $2500 \mathrm{~mL}$ ácido fosfórico marcado con ${ }^{32} \mathrm{P}$, con una actividad de $0,144 \mathrm{mCi} / \mathrm{mL}^{-1}$, aplicando una actividad de $8,64 \mathrm{mCi}$ por árbol con una dosis de $5 \mathrm{~mL}$ por hueco. En total se utilizó un análisis de fertilidad de suelo y foliar para determinar el estado nutricional de los árboles.

Los muestreos de estructuras (hoja, tallo y fruto) se realizaron cada 15 días para un total de 6 muestreos. Las hojas muestreadas eran de desarrollo intermedio, los tallos de la parte media de ramas productivas y los frutos con tres cuartos de maduración. Las muestras se analizaron en el laboratorio de nutrición vegetal del Instituto de Ciencias Nucleares y Energías Alternativas (INEA) en Bogotá. Los tejidos colectados se secaron en una estufa a una temperatura de $70^{\circ} \mathrm{C}$ hasta peso seco constante y de esta muestra se tomaron $5 \mathrm{~g}$, los que se calcinaron en una mufla a $600^{\circ} \mathrm{C}$ durante ocho horas. Las cenizas obtenidas se disuelven una solución de $\mathrm{HCl}$ y $\mathrm{HNO}_{3}$. $\mathrm{La}$ actividad se determinó en un contador de centelleo líquido por el método de Cerenkov.

La fórmula para el cálculo de la actividad fue:

$$
\begin{aligned}
& A=\text { Actividad }=A_{0} \text { exp. }-0,693 t / T^{1 / 2} \\
& A=\text { Actividad al tiempo } t \\
& A 0=\text { Actividad al tiempo cero } \\
& t=\text { Tiempo desde la aplicación del radioisótopo } \\
& T 1 / 2=\text { Tiempo de vida media del radioisótopo en días }
\end{aligned}
$$


Tabla 1. Características físicas, químicas, suelos Tipic haplustalf en el sitio experimental.

Flandes, Tolima (según Castro, 1996).

\begin{tabular}{|c|c|c|c|c|c|c|c|c|c|c|c|c|c|}
\hline Profundidad & Textura & pH & $\%$ & \multicolumn{4}{|c|}{ meq/100 g } & \multicolumn{7}{c|}{ ppm } \\
\hline \multicolumn{3}{|c|}{} & M.0 & Ca & Mg & K & Na & P & B & Cu & Fe & Mn & Zn \\
\hline $0-30$ & F.A & 6,3 & 0,85 & 4,9 & 1,7 & 0,42 & 0,45 & 30,1 & 0,29 & 1,8 & 20,6 & 3,4 & 1,3 \\
\hline
\end{tabular}

La actividad en \% se determinó por la fórmula:

$$
\begin{aligned}
& \begin{array}{l}
\text { Porcentaje actividad radical para } \\
\text { una distancia y profundidad }
\end{array}=\frac{\mathrm{cpm} / \mathrm{g} \text { material para el tratamiento } \times 100}{\mathrm{cpm} \text { totales } / 7 \mathrm{~g} \text { material muestreado para }} \\
& \text { todos los tratamientos }
\end{aligned}
$$

\section{RESULTADOS Y DISCUSIÓN}

Primer muestreo: (15 días). Para este muestreo se presentaron diferencias significativas entre los tratamientos, entre distancia de aplicación no hubo diferencias significativas, sin embargo, la de $150 \mathrm{~cm}$ mostró el mayor valor. Se presentaron diferencias entre profundidades siendo mayor la actividad a $30 \mathrm{~cm}$ con una actividad promedio de $14,42 \%$. La estructura donde más se detectó la absorción de ${ }^{32} \mathrm{P}$ correspondió a la hoja, seguida del tallo. Se presentó una interacción positiva y significativa entre distancia y profundidad.

Segundo muestreo: (30 días). A los 30 días de haberse aplicado los tratamientos no existieron diferencias entre las distancias y tampoco en la actividad a diferentes profundidades. Sin embargo, la tendencia es de una mayor actividad a $100 \mathrm{~cm}$ y a una profundidad de $30 \mathrm{~cm}$. La mayor absorción de ${ }^{32} \mathrm{P}$ correspondió a la hoja y en segundo lugar al tallo. No hubo diferencias significativas entre distancia y profundidad.

Tercer muestreo: (45 días). A los 45 días se presentaron diferencias significativas en la actividad entre las distancias de aplicación del isótopo siendo la mejor la de $100 \mathrm{~cm}$ entre 150 y 200 no hubo diferencias. La mejor actividad se detectó a los $15 \mathrm{~cm}$ de profundidad. La estructura de mayor absorción fue la hoja, el tallo y el fruto respectivamente. Se presentó una interacción positiva y significativa al $6 \%$ entre distancia y profundidad.

Cuarto muestreo: (60 días). A los 60 días de la aplicación se presentaron diferencias altamente significativas entre los tratamientos, presentándose la mayor actividad a una distancia de 100 $\mathrm{cm}$, seguida por la de $150 \mathrm{~cm}$. En profundidad la mayor actividad se detectó a los $15 \mathrm{~cm}$. La estructura de mayor absorción del isótopo continuó siendo la hoja. Se presentó una interacción positiva y altamente significativa entre la distancia y la profundidad.

Quinto muestreo: (75 días). En este muestreo se presentaron diferencias significativas en la actividad radical de las plantas evaluadas. La distancia donde se muestre la mayor actividad $(18,22 \%)$ fue a $100 \mathrm{~cm}$, seguida por la de $150 \mathrm{~cm}$, no encontrándose diferencias entre ellas pero sí con la de $200 \mathrm{~cm}$. La mejor profundidad fue la de $15 \mathrm{~cm}$, seguido por la de 30 y $45 \mathrm{~cm}$ no encontrándose diferencias entre estas. No se encontraron diferencias en las tasa de absorción del ${ }^{32} \mathrm{P}$ para cada una de las estructuras muestreadas, pero continuó siendo la hoja la de mayor absorción. No se presentaron diferencias en la interacción distancia x profundidad. 
Sexto muestreo: (90 días). A los 90 días posteriores a la aplicación se encontraron diferencias entre la actividad radical de los árboles. No hubo diferencias entre las distancias, pero sí entre las profundidades, siendo la de $15 \mathrm{~cm}$ la mayor $(18,25 \%)$ diferente significativamente con las profundidades de 45 y $30 \mathrm{~cm}$ respectivamente.

Para esta época el órgano de mayor absorción fue el tallo seguido por las hojas y finalmente el fruto, aunque no existieron diferencias significativas entre ellos. La actividad en tallo y hoja se muestra en la figura 1 y 2.
El comportamiento de la actividad para las diferentes profundidades de aplicación del isótopo se muestra en la figura 3.

En la figura 4 se observa el comportamiento de la actividad para las tres distancias de aplicación evaluadas.

La absorción del ${ }^{32} \mathrm{P}$ fue diferente entre las estructuras muestreadas siendo mayor $(18,01 \%)$ en el tallo, en segundo lugar la hoja (17,99\%) y por último la menor detección del ${ }^{32} \mathrm{P}$ fue en el fruto $(17,61 \%)$.

Tabla 2. Resultados de actividad radical de plantas de limón, muestreo de hojas (promedio de cuatro repeticiones). ${ }^{32} \mathrm{P}$ recuperado en cuentas por minuto $\mathrm{cpm}$.

\begin{tabular}{|c|c|c|c|c|c|}
\hline Intervalo de muestreo (días) & Profundidad (cm) & \multicolumn{3}{|c|}{ Distancia (cm) } & Promedio profundidades \\
\hline \multirow{4}{*}{15} & & 100 & 150 & 200 & \\
\hline & 15 & 15,05 & 14,19 & 13,72 & 14,32 \\
\hline & 30 & 14,79 & 15,48 & 13,11 & 14,16 \\
\hline & 45 & 13,43 & 13,88 & 14,54 & 13,95 \\
\hline Promedio distancia & & 14,42 & 14,51 & 13,79 & \\
\hline \multirow{3}{*}{30} & 15 & 16,64 & 16,99 & 16,21 & 16,61 \\
\hline & 30 & 15,99 & 16,09 & 15,82 & 15,96 \\
\hline & 45 & 15,80 & 15,88 & 16,61 & 16,09 \\
\hline Promedio distancia & & 16,14 & 16,32 & 16,21 & \\
\hline \multirow{3}{*}{45} & 15 & 17,62 & 17,25 & 16,79 & 17,22 \\
\hline & 30 & 17,42 & 16,86 & 16,49 & 16,92 \\
\hline & 45 & 16,84 & 15,94 & 16,71 & 16,49 \\
\hline Promedio distancia & & 17,29 & 16,68 & 16,66 & \\
\hline \multirow{3}{*}{60} & 15 & 17,88 & 17,62 & 16,94 & 17,48 \\
\hline & 30 & 18,06 & 17,34 & 16,32 & 17,24 \\
\hline & 45 & 17,09 & 17,08 & 17,87 & 17,34 \\
\hline Promedio distancia & & 17,67 & 17,34 & 17,04 & \\
\hline \multirow{3}{*}{75} & 15 & 18,22 & 18,06 & 17,43 & 17,90 \\
\hline & 30 & 17,79 & 17,67 & 17,31 & 17,59 \\
\hline & 45 & 17,05 & 17,01 & 17,17 & 17,07 \\
\hline Promedio distancia & & 17,68 & 17,58 & 17,30 & \\
\hline \multirow{3}{*}{90} & 15 & 18,25 & 18,46 & 18,53 & 18,41 \\
\hline & 30 & 17,70 & 17,18 & 17,33 & 17,40 \\
\hline & 45 & 17,72 & 17,80 & 17,87 & 17,79 \\
\hline Promedio distancia & & 17,89 & 17,81 & 17,91 & \\
\hline
\end{tabular}


Los resultados mostraron que las raíces fueron más activas en la toma y absorción del trazador en todas las distancias laterales y en todas las profundidades en las cuales fue aplicado. Generalmente se observó que la toma de ${ }^{32} \mathrm{P}$ fue mayor a $100 \mathrm{~cm}$ de distancia y en los primeros $15 \mathrm{~cm}$ de profundidad, hecho que se pudo comprobar en los muestreos de hojas y tallo, lo que significa que el mayor actividad del sistema radical de la lima ácida se encuentra en el horizonte superficial del suelo, coincidiendo con especies frutales injertados sobre patrones clonales (Fischer, 1994).

Después del cuarto muestreo la actividad empezó a disminuir a esta distancia pero se incrementó entre los 100 y $150 \mathrm{~cm}$ de distancia y en los primeros $15 \mathrm{~cm}$ de profundidad, lo que indica que para árboles de limón común de 5 años de edad, las raíces normales más activas se encuentran en los primeros $15 \mathrm{~cm}$ de profundidad del suelo a una distancia del tronco entre 100 y $150 \mathrm{~cm}$, mientras que en manzano, Friedrich et al. (1986) reportaron la mayor cantidad de puntas radicales en los primeros $20 \mathrm{~cm}$ del suelo.

El hecho de que existan allí las raíces de mayor actividad indica que para las condiciones de suelo mencionadas y para las condiciones de este experimento el suministro de agua y nutrientes para el cultivo debe hacerse entre 100 y $150 \mathrm{~cm}$ de distancia del tronco, bien distribuidos y a una profundidad no mayor a los $15 \mathrm{~cm}$. El comportamiento observado de absorción también mostró que el decrecimiento en la actividad en general está ligada al aumento de la profundidad y al aumento de la distancia lateral. La toma del ${ }^{32} \mathrm{P}$ fue mínima por encima de los $30 \mathrm{~cm}$ de profundidad. El incremento de la actividad a partir de los 75 días en la distancia dos podría deberse al incremento del contenido de humedad de suelo.

Debido a que la mayor absorción de ${ }^{32} \mathrm{P}$ correspondió a la cantidad de este elemento en la hoja, se confirma lo expresado por Persson (2002) que

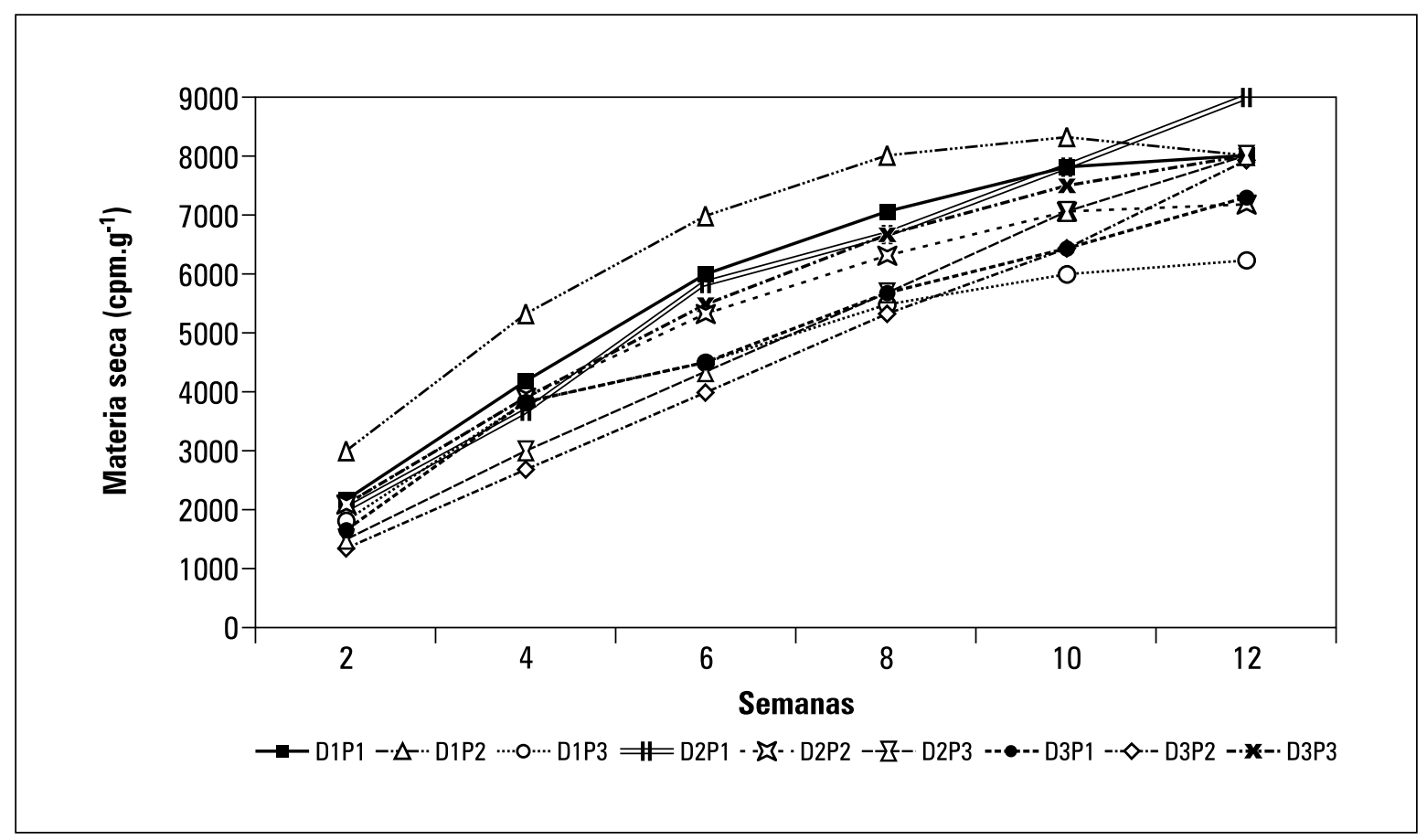

Figura 1. Comportamiento de la actividad radical en lima ácida mexicana a diferentes distancias D1: 100 cm, D2: $150 \mathrm{~cm}$, D3: $200 \mathrm{~cm}$ y profundidades P1: $15 \mathrm{~cm}, \mathrm{P2}: 30 \mathrm{~cm}$ y P3: $45 \mathrm{~cm}$. Muestreo en tallos. 


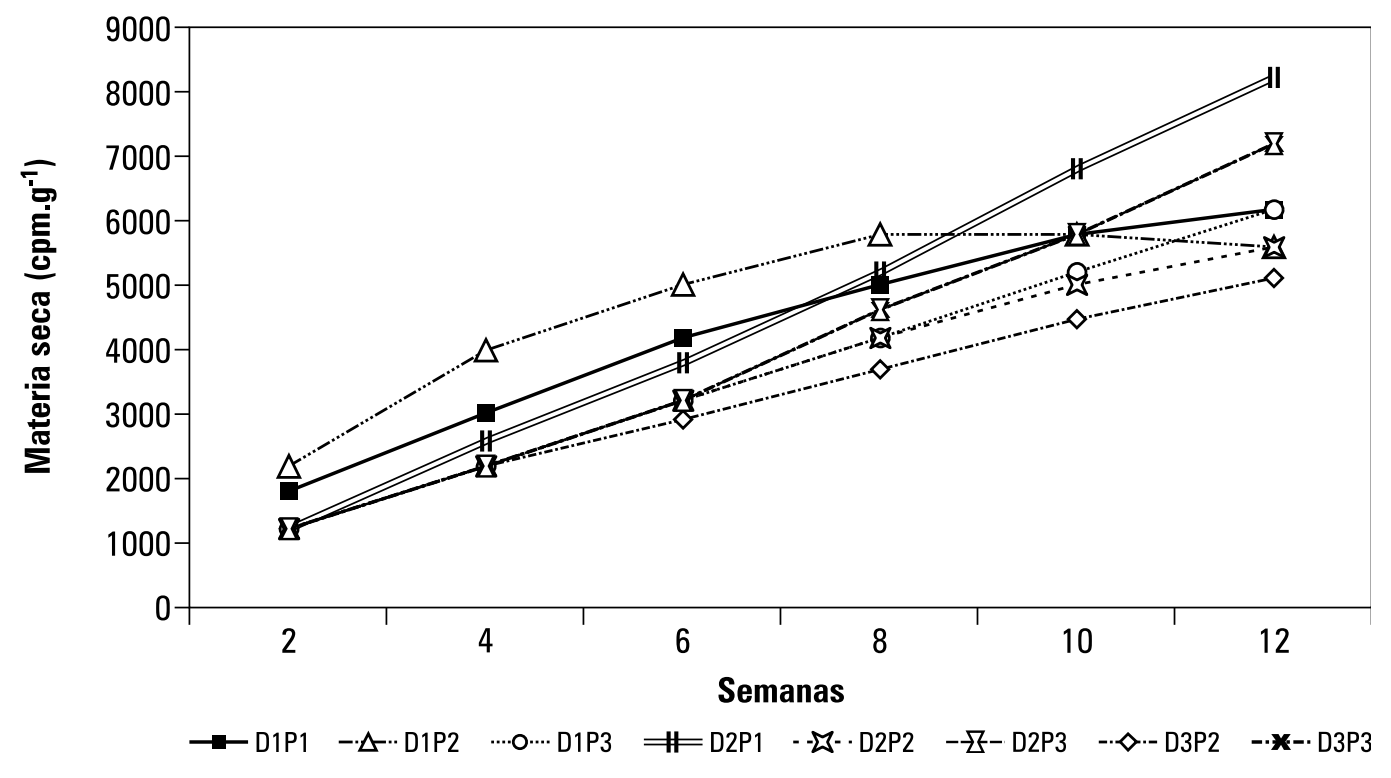

Figura 2. Comportamiento de la actividad radical en lima ácida mexicana a diferentes distancias D1: 100 cm, D2: $150 \mathrm{~cm}$, D3: $200 \mathrm{~cm}$ y profundidades P1: $15 \mathrm{~cm}$, P2: $30 \mathrm{~cm}$ y P3: $45 \mathrm{~cm}$. Muestreo en hojas.

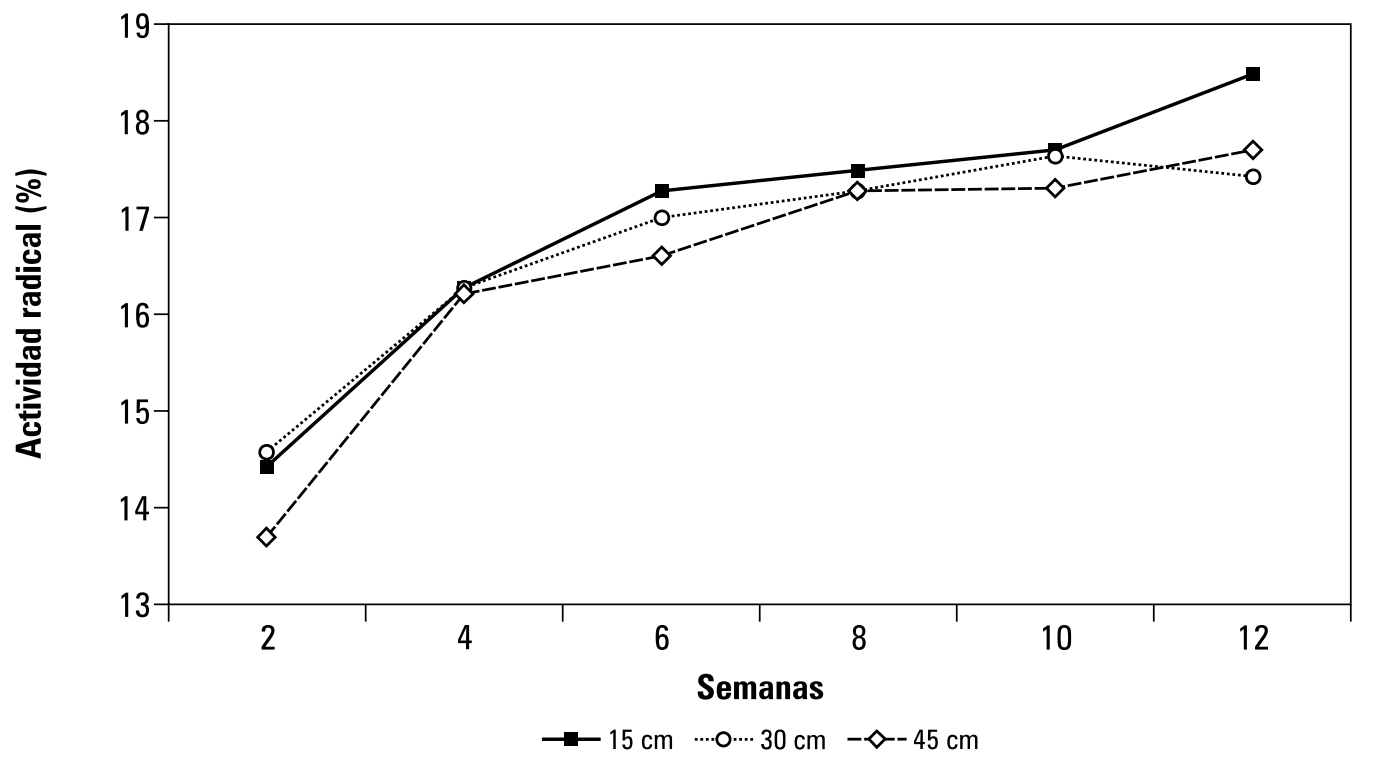

Figura 3. Comportamiento de la actividad radical en lima ácida mexicana a diferentes profundidades de aplicación del isótopo ${ }^{32} \mathrm{P}$. 


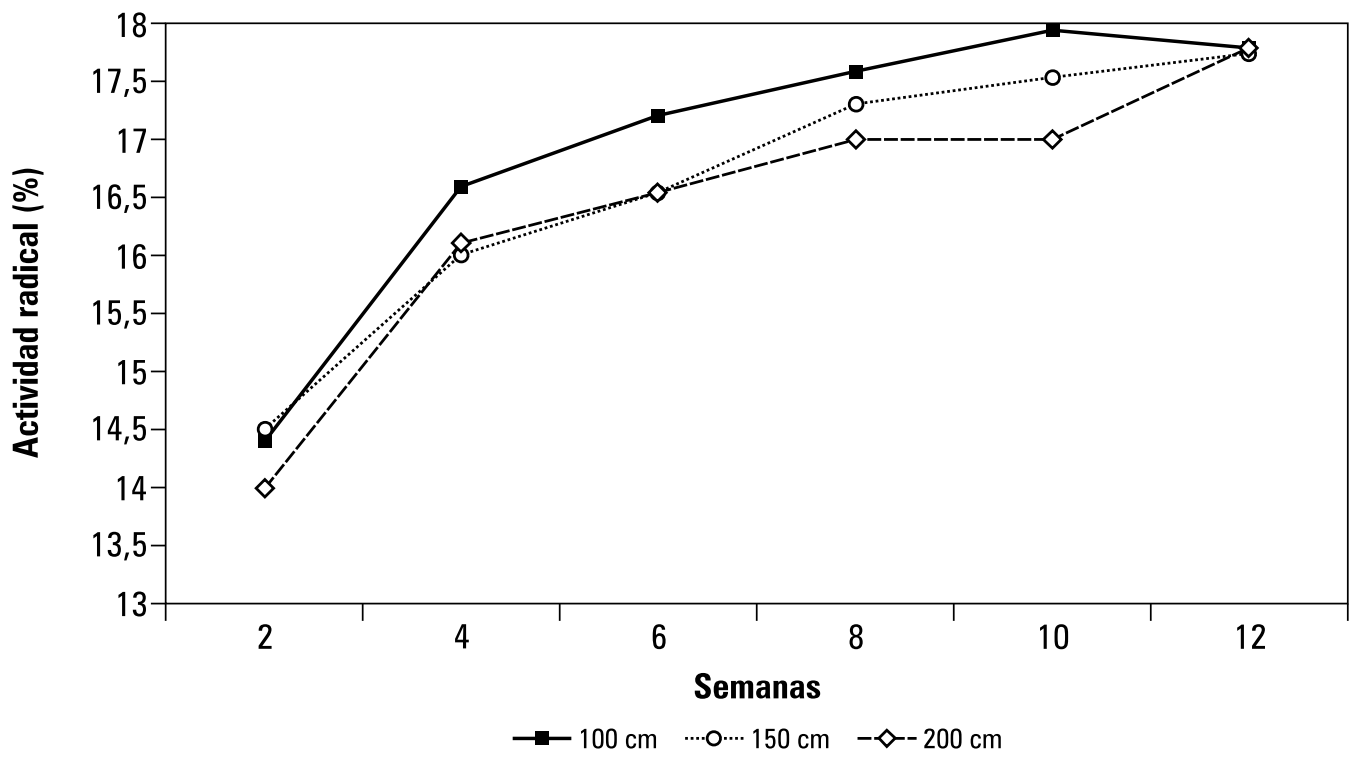

Figura 4. Comportamiento de la actividad radical en lima ácida mexicana a diferentes distancias de aplicación del isótopo ${ }^{32} \mathrm{P}$.

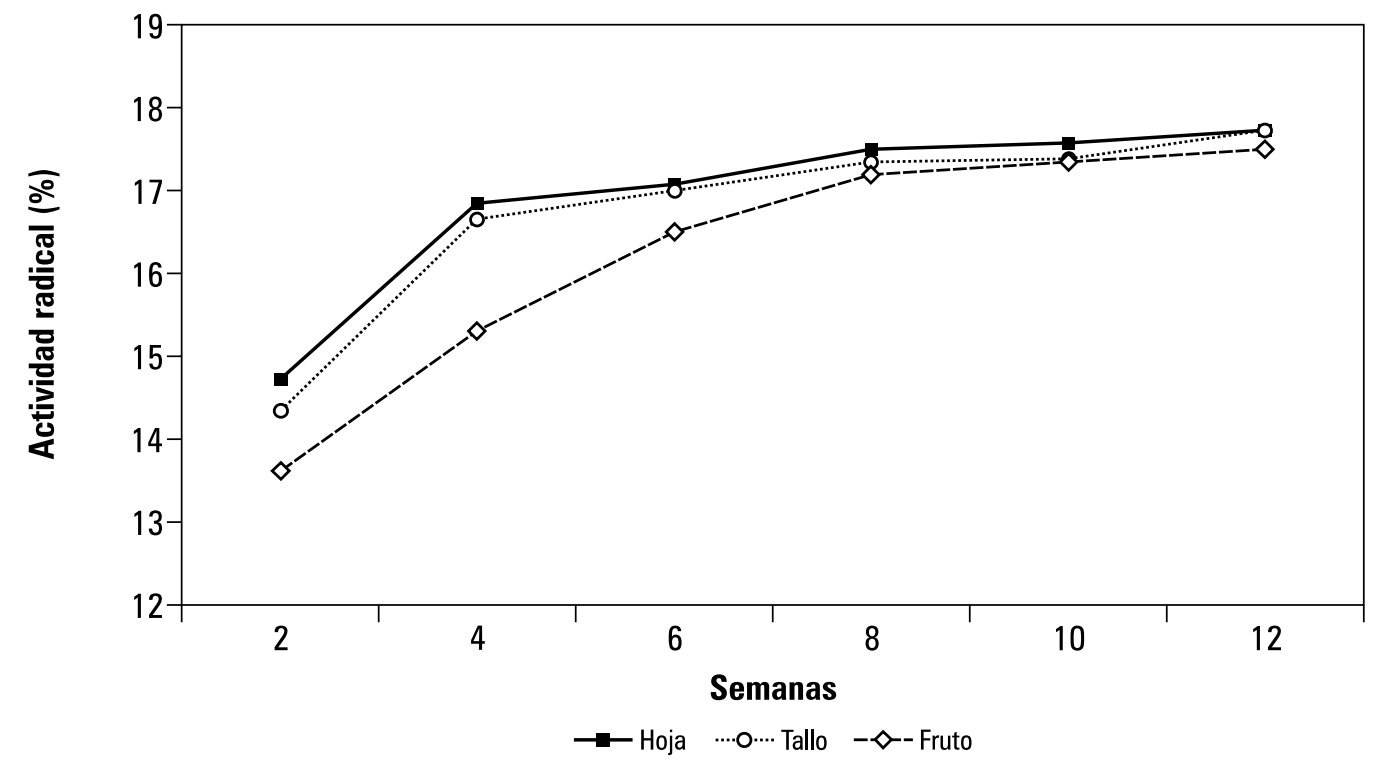

Figura 5. Comportamiento de la actividad radical en lima ácida mexicana según estructura de la planta muestreada. 
existe un equilibrio funcional entre tamaño y actividad de las hojas (que fijan carbono), el tamaño y actividad de las raíces finas (que toman nutrientes y agua).

De acuerdo con los resultados, en el área de mayor actividad radical no se deben ubicar cultivos como arreglos múltiples (intercalados), tampoco realizar labores de desyerba profundas en esta área para evitar daño de las raíces.

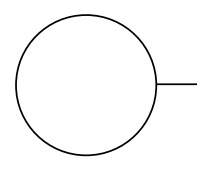

\section{REFERENCIAS BIBLIOGRÁFICAS}

Agusti, M. 2003. Citricultura. 2da. edición. Ediciones Mundi-Prensa, Madrid. 422 p.

Bujan, A.; M.B Barrios; A. Bozzo; S.P. Debelis y M.S. Yáñez. 1998. Empleo de técnicas nucleares para el estudio del desarrollo radicular en soja (Glycine max). En: http://www.cab.cnea.gov.ar/AATN99/Actas/Docs/R4I65.pdf; consulta: enero 2007.

Castro, H.E. 1996. Bases técnicas para el conocimiento y manejo de los suelos del valle cálido del alto magdalena. Corpoica regional 6. Podumedios. Bogota. 107p.

Corporación Colombia Internacional. 2002. Limas y limones. Perfil del producto. No. 18. Ministerio de Agricultura y Desarrollo Rural, Bogotá. 12 p.

Delgado, C. 1995. Evaluación de la actividad radical en Palma Africana (Eleais guineensis Jacq.) utilizando fósforo-32. Trabajo de grado. Facultad de Agronomía, Universidad Nacional de Colombia, Bogotá. 65 p.

Epstein, E. y A.J. Bloom. 2005. Mineral nutrition of plants: principles and perspectives. $2^{\text {nd }}$ edition. Sinauer Associates, Inc. Publishers, Sunderland, Massachusetts. 400 p.

Fischer, G. 1994. Crecimiento y desarrollo de los frutales caducifolios. Programa de Posgrado, Facultad de Ciencias Agropecuarias, UPTC Tunja. 89 p.
International Atomic Energy Agency. 1975. Root activity patterns of some tree crops. Technical Reports Serie $\mathrm{N}^{\circ} 170$. Viena.

López, S.; N. Bárbaro; S. Rojas; M. Melaj; J.P. Bonetto; O. Martín y A. Solís. 2000. Aporte de las técnicas isotópicas en las recomendaciones de uso de fertilizantes y la conservación del recurso suelo. Unidad de actividad aplicaciones tecnológicas y agropecuarias. Centro Atómico Ezeiza. Comisión Nacional de Energía Atómica. 4 p.

Persson, H.A. 2002. Root systems of arboreal plants. En: Waisel, Y.; A. Eshel y L. Kafkafi (eds.). Plant roots - The hidden half. 3rd edition. Marcel Dekker, New York. pp. 187-2004.

Pino, I. 2005. Técnicas isotópicas en estudio de suelos. En: http//mazinger.sisib.uchile.cl/repositorio/lb/ciencias agronomicas/miscelaneasagronomicas38/C15. htmi ; consulta: enero 2007.

Salisbury, F.B. y C.W. Ross. 1994. Fisiología vegetal. Grupo Editorial Iberoamérica, México. 759 p.

Ryugo, K. 1993. Fruticultura - ciencia y arte. AGT Editor, México. 460 p. 